\title{
Clinical and Biological Concepts for Mastering Immune Reconstitution After HSCT: Toward Practical Guidelines and Greater Harmonization
}

\author{
Jürgen Kuball and Jaap Jan Boelens
}

\subsection{Introduction/Background}

The main mechanisms of action resulting in a long-term cure, but also in many life-threatening side effects after HSCT, are mediated by the rapidly reconstituting immune repertoire, which depends on the conditioning regimen, cell dose and graft composition, as well as the type of immune suppression. However, knowledge of these mechanisms is limited, due to many variations in clinical programs, including the specific type of transplantation procedure, as well as a lack of standardized immune monitoring after HSCT.

To date, only the process of donor selection has been significantly impacted by new biological insights, but little attention has been given to the design of the cell product in terms of numbers and composition, to avoid variations between different patients. In addition, high variations between patients in the clearance of agents used

\section{J. Kuball $(\bowtie)$ \\ Department of Hematology, UMC, Utrecht, The Netherlands}

Laboratory of Translational Immunology, UMC, Utrecht, The Netherlands e-mail: J.H.E.Kuball@umcutrecht.nl

\section{J. J. Boelens}

Laboratory of Translational Immunology, UMC, Utrecht, The Netherlands

Memorial Sloan Kettering Cancer Center, New York, NY, USA during the conditioning are rarely investigated. Given the dearth of prospective clinical studies addressing these important concepts, and the fact that such studies will most likely never be performed, due to the lack of interest from pharmaceutical companies, we aim to initiate a consensus discussion. Our goal is to harmonize the intervention HSCT by exploring how individual differences between patients and overall transplantation strategies impact the final effector mechanisms of HSCT, namely, a timely and wellbalanced immune reconstitution.

\subsection{Impact of Conditioning Regimens on Immune Reconstitution and Outcomes: Pharmacokinetics- Pharmacodynamics (PK-PD), Individualized Dosing}

Various groups have recently demonstrated that agents administered as part of the conditioning regimen, as well as after HSCT, will influence both short-term and long-term immune reconstitution (Soiffer and Chen 2017; Admiraal et al. 2015). These agents may, therefore, have an unknown effect on also other cell-based therapeutics. In the context of HSCT, "predictable" immune reconstitution is important when studying maintenance therapies with novel drugs, DLI, 
and advanced cell therapy interventions. Therefore, it is essential to understand the impact of the agents used on the immune reconstitution. Comprehensive pharmacokinetic (PK) and pharmacodynamic (PD) information can help to illuminate the effects that exposure of agents in the conditioning have on immune reconstitution and subsequent outcomes (e.g., GvHD, relapse and non-relapse mortality).

The recent discovery that the pharmacokinetics of serotherapy (e.g., ATG and ATLG) is highly dependent on receptor load (represented by absolute lymphocyte count; ALC) before the first dosing is one example. In adults, receptor load was the only predictor for ATG clearance, while in pediatric patients $(<40 \mathrm{~kg})$, weight also influenced clearance. While prospective validation trials of novel ATG nomograms currently include patients linked to defined transplantation regimens, initial recommendations for dosing serotherapy on lymphocyte count rather than body weight seem to be reasonable, e.g., within the context of $\mathrm{T}$ cell-replete reduced conditioning regimens (Admiraal et al. 2015) (Table 10.1). From a post hoc analysis of a recent randomized controlled trial allowing three different types of regimens, we learned that different regimens had the reverse effects of ATLG on the outcomes, resulting in overlapping curves for the primary endpoint, chronic-GvHD-free, leukemia-free survival (Soiffer et al. 2017).

Table 10.1 Suggested novel ATG dosing nomograms based on PK-PD modeling for (non-)myelo-ablative settings in pediatrics and adults ${ }^{\mathrm{a}}$

\begin{tabular}{l|l|l|l|} 
& $\begin{array}{l}\text { Dosing } \\
\text { on }\end{array}$ & $\begin{array}{l}\text { Target AUC after } \\
\text { HSCT (AU*d/mL) } \\
\text { and donor source }\end{array}$ & $\begin{array}{l}\text { Starting } \\
\text { day }\end{array}$ \\
\hline $\begin{array}{l}\text { Setting } \\
\text { Pediatrics; } \\
\text { MAC Admiraal } \\
\text { et al. (2015) }\end{array}$ & $\begin{array}{l}\text { Weight } \\
\text { ALC }\end{array}$ & $\begin{array}{l}<20 \text { for cord blood } \\
<50 \text { for bone } \\
\text { marrow }\end{array}$ & 9 \\
\hline $\begin{array}{l}\text { Adults: } \\
\text { source }\end{array}$ & ALC & $\begin{array}{l}\text { 60-90 for } \\
\text { peripherally }\end{array}$ & 9 \\
$\begin{array}{l}\text { Admiraal et al. } \\
\text { (2017) }\end{array}$ & & $\begin{array}{l}\text { mobilized stem } \\
\text { cells }\end{array}$ & \\
\hline
\end{tabular}

$A L C$ absolute lymphocyte count, $A U C$ area under the curve

${ }^{\mathrm{a}}$ Level C evidence (retrospective studies)
Serotherapy is not the only agent in a conditioning regimen with variable PK that can have a dramatic impact on the chances for survival. In a recent retrospective cohort analysis that included more than 650 pediatric and young adult patients, cumulative exposure to BU was found to influence outcomes (Bartelink et al. 2016). The optimal BU exposure, for the main outcome of EFS, was found to be independent of indication, combination (BU/FLU, BU/CY, or BU/CY/MEL), age, and donor source. BU/FLU within the optimal BU exposure $(80-100 \mathrm{mg} * \mathrm{~h} / \mathrm{L})$ was associated with the highest survival chances and lowest toxicity compared to other combinations. More recently, fludarabine exposure was also found to influence survival (in an ATG-FLU/BU: Boelens et al. 2018). These studies further illustrate that pharmacokinetic variations in individuals can have significant effects on survival. Historically, and still in daily practice, a variety of conditioning regimens are used, which complicates comparisons of HSCT outcomes across different centers and even within trials.

\subsection{Graft Composition as an Additional Predictor for Immune Reconstitution and Clinical Outcomes}

Although transplant physicians carefully monitor the levels of many drugs, such as CSA or antibiotics, an additional opportunity to further harmonize the transplantation procedure arises from the surprising clinical observation that substantial cell dose variations are currently accepted across patients. The hesitation to monitor cell numbers in the graft or after HSCT, and to act on them, is of course partially driven by the confusing magnitude of immunological subsets, the narrow nature of many immunological programs with a lack of consensus on immune monitoring, and also rather limited immunological education across the majority of transplant physicians. However, currently available retrospective and prospective studies can provide guidance. A retrospective EBMT study indicated that $\mathrm{T}$ cell numbers vary frequently between 50 and 
$885 \times 10^{6} / \mathrm{kg}$ and the highest quartile in CD34+ cells, as well as $\mathrm{T}$ cells associate with an inferior clinical outcome (5). As we cannot expect in the future randomized trials addressing the impact of different graft compositions in $\mathrm{T}$ cell-replete transplantations on clinical outcomes, avoiding higher numbers of CD34 and T cells within the highest quartile might be reasonable (Czerw et al. 2016). Higher numbers of NKT cells (Malard et al. 2016) and $\gamma \delta T$ cells (Perko et al. 2015) in the graft have been reported to associate with favorable immune reconstitution, and a positive clinical outcome, most likely due to their impact on controlling GVHD (Du et al. 2017) and acting on CMV, as well as on leukemia (Scheper et al. 2013; de Witte et al. 2018). However, these variables are more difficult to control in daily clinical practice. Direct ex vivo graft engineering provides an elegant solution to further control immune subsets in the graft and the consecutive immune reconstitution. It also allows for the standardization of cell numbers, as well as subsets per patient, e.g., selecting CD34positive stem cells alone has been reported to associate with less chronic GVHD, while the graft versus leukemia effect is maintained (Pasquini et al. 2012). As the next generation of graft engineering, depletion of $\alpha \beta T$ cells has been reported to associate with lower frequencies of infection and very low GVHD rates (Locatelli et al. 2017).

\subsection{Immune Monitoring}

\subsubsection{Immune Cell Phenotyping}

The most important questions that arise when monitoring immune therapeutic interventions are:

1. How many cells within each leukocyte subset are present in patients at different stages of disease, before immune intervention?

2. What is the immune composition of the graft?

3. Which immune subsets are reconstituting at what points in time?
4. What is the functional response of these cells to additional immunotherapeutic or drug interventions after transplantation (Table 10.1)?

These questions are particularly important in an era when post-HSCT pharmaceutical maintenance interventions and DLI or the administration of other ATMPs (advanced therapy medicinal products) have become daily practice for many different disease categories (Soiffer and Chen 2017).

Flow cytometry is often available for comprehensive immune phenotyping, usually in accredited laboratories within transplant centers. Markers identifying the most common leukocyte subsets are broadly used and can therefore be considered as a "standard" panel: CD45 (lymphocytes), CD3 (T cells), CD19 (B cells), $\alpha \beta T C R, \gamma \delta T C R$, and CD16/CD56 (NK) cells. In some centers/studies, this panel has been extended to identify the differentiation and activation state of subsets of $\mathrm{T}$ ( $\mathrm{T}$-helper, regulatory T cells), B, and NK(T) cells, as well as cells from the myeloid lineage (monocytes, dendritic cell subsets). This knowledge is important because the success of cell-based immunotherapies, as well as agents modulating the immune system after transplantation, will significantly depend on the presence or absence of different immune subsets. Mastering the diversity might allow for the definition of subpopulations who would benefit from checkpoint-inhibitor treatment after HSCT, as well as characterize patients who would be at high risk for GVHD, while currently this intervention is considered to be very toxic (Davids et al. 2016). Also, other subsets may be suitable as biomarkers to predict clinical efficacy. Given the potential impact of sorafenib on post-HSCT outcomes through the induction of IL15 (Mathew et al. 2018), additional immune subsets associating with improved leukemia control need to be identified. In another study, high baseline frequencies of peripheral blood dendritic cells (DC) correlated with a clinical response to high-dose IL-2 (Finkelstein et al. 2010). These data emphasize the importance of DC in endogenous and therapy-induced antitumor immunity and arguably warrant the incorporation of DC markers in immune-monitoring panels. 
Taken together, a variety of specialized subsets may have potential as predictive markers for clinical efficacy, but they require more sophisticated staining protocols, making more cumbersome staining techniques less broadly applicable for harmonized panels across centers or in multicenter clinical trials. Furthermore, it is important to note that trials using whole blood assays may produce different percentages of cell subsets when compared with studies using PBMCs. The same is true when comparing freshly isolated PBMCs with biobanked material, which has been subjected to freeze/thaw procedures that affect expression levels of various markers. Even when the same samples are collected, variations can be introduced by the selection of antibody clones, combination of clones and fluorochromes, and the gating strategies. In sum, minimizing the variability in sample handling and the preanalysis phase is critical for standardization.

\subsubsection{Immune Monitoring: Secretome Analyses}

Measuring the production of cytokines, chemokines, and growth factors and their profiles (i.e., the secretome) represents an integral part of immunomonitoring during immunotherapeutic treatments. These biomarkers may distinguish diverse disease/response patterns, identify surrogate markers of efficacy, and provide additional insight into the therapeutic mode of action. Peripheral blood is often the only source for protein analysis, which may lack the sensitivity to reflect local responses in affected tissues. As examples, proteins, such as interleukin-6, granulocyte-macrophage colony-stimulating factor (GM-CSF), hepatocyte growth factor (HGF), ST2 (suppressor of tumorigenicity), and soluble IL-2a, have been suggested as potential biomarkers for GvHD, whereas increased levels of TNF-a and IL-6 are associated with robust immune responses to viral reactivation (de Koning et al. 2016).

The most commonly used methods to identify these markers include antibody-based ELISA or multiplex platforms, such as protein microarrays, liquid chromatography-mass spectrometry (LCMS), electro-chemiluminescence, and beadbased multiplex immunoassays (MIA). Again, different technologies and reagents (e.g., antibodies and recombinants for standard curves) may lead to different concentrations and dramatic variability in results, depending on how the preanalytic samples are handled (e.g., differences in processing and storage, including duration of storage). Cytokine levels differ considerably between serum and plasma samples obtained from the same donor, due to release of plateletassociated molecules into serum. Moreover, the type of anticoagulant used in plasma isolation and time- and/or temperature-sensitive changes need to be considered (Keustermans et al. 2013). These phenomena underscore the need for extensive documentation with respect to all biomarker analysis before any conclusions can be made when comparing patient cohorts treated at multiple sites.

\subsection{Summary}

The failure or success of HSCT is significantly impacted by the patient's immune status. However, only a minority of HSCT programs systematically consider individualized drug monitoring during conditioning, graft design, and immune monitoring as key for patient surveillance, in order to maximally control and capture essential details of the intervention HSCT. Therefore, guidelines are needed to further harmonize the procedure HSCT as well as standardized immune monitoring to allow for distillation of key features for success and failure. First, careful recommendations for individualized drug dosing as well as graft compositions can be made based on available data sets. However, it will be key to register within the new cellular therapy registry of EBMT additional details of drug dosages, graft compositions, as well as immune reconstitution, to capture clinical variations in programs, as well as defined immune reconstitutions. This will enable a retrospective 
Table 10.2 Panels under consideration in the panel discussion of the CTIWP (Greco et al. 2018) ${ }^{\mathrm{a}}$

\begin{tabular}{|c|c|c|c|}
\hline & \multicolumn{2}{|l|}{ General } & Advanced \\
\hline Graft composition & $\begin{array}{l}\alpha \beta \mathrm{T} \\
\gamma \delta \mathrm{T} \\
\text { Treg } \\
\text { B } \\
\text { NK/NKT }\end{array}$ & $\begin{array}{l}\alpha \beta T C R, C D 45 R O / R A, C D 3, C D 4, \\
\text { CD8, CD27 } \\
\gamma \delta T C R, C D 45 R O / R A, C D 3, \text { CD27 } \\
\text { CD } 45, \text { CD } 4, \text { CD25, CD127, FoxP3 } \\
\text { CD45, CD19, CD38, CD27, } \\
\text { IgM/G/D, CD21 } \\
\text { CD45, CD3, CD56, TCR } \alpha 24 / \beta 11)\end{array}$ & $\begin{array}{l}\text { Intracellular cytokines } \\
\text { after PMA/ionomycin stimulation } \\
\text { Specific TCR by multimer } \\
\text { approach }\end{array}$ \\
\hline $\begin{array}{l}\text { Cell phenotyping } \\
\text { pre- and post } \\
\text { transplantation }\end{array}$ & $\begin{array}{l}\alpha \beta \mathrm{T} \\
\gamma \delta \mathrm{T} \\
\text { Treg } \\
\mathrm{B} \\
\text { NK/NKT } \\
\text { DC/mono }\end{array}$ & $\begin{array}{l}\alpha \beta T C R, C D 45 R O / R A, C D 3, C D 4, \\
\text { CD8, CD27 } \\
\gamma \delta T C R, C D 45 R O / R A, C D 3, \text { CD27 } \\
\text { CD } 45, \text { CD } 4, \text { CD25, CD127, FoxP3 } \\
\text { CD45, CD19, CD38, CD27, } \\
\text { IgM/G/D, CD21 } \\
\text { CD45, CD3, CD56, TCR } 24 / \beta 11) \\
\text { CD11c, HLA-DR, CD14, CD16, } \\
\text { CD1c, CD141, CD303 }\end{array}$ & $\begin{array}{l}\text { Intracellular cytokines } \\
\text { after PMA/ionomycin stimulation } \\
\text { Specific TCR by multimer } \\
\text { approach } \\
\alpha \beta T C R \text { and } \gamma \delta T C R \text { repertoire }\end{array}$ \\
\hline Secretome & - & & $\begin{array}{l}\text { Multiplex panel (e.g., IL-7, ST2, } \\
\text { TNF-a, IL-6, HGF, IL-2R, IL-8, } \\
\text { GM-CSF, etc.) }\end{array}$ \\
\hline Cell function & - & & $\begin{array}{l}\text { NK cell lyses } \\
\mathrm{T} \text { cell proliferation upon antigens } \\
\text { and mitogens } \\
\mathrm{B} \text { cell maturation }\end{array}$ \\
\hline PK & BU, FLU, & Campath (if part of conditioning) & Trial drug \\
\hline MRD & qPCR (tar & pressed, flow cytometry) & Next-generation sequencing \\
\hline Viral load & CMV, EB & , adenovirus & - \\
\hline
\end{tabular}

increase in insight into daily clinical practice, and its impact on immune reconstitution, as well as clinical outcome. Also, clinical trials should adopt such consensus measurements. Nevertheless, the markers and phenotypes studied in one setting may not be considered relevant in another, supporting the definition of a set of general recommended protocols and a set of addon trial-specific parameters (Table 10.2). A consensus panel is currently prepared by the cellular therapy and immunobiology working party (CTIWP) of EBMT (Greco et al. 2018). A harmonization procedure to achieve a more balanced immune reconstitution might have a more profound impact on patient survival than any other novel maintenance therapy (Admiraal et al. 2017; Boelens et al. 2018) and allow for a better success rate for novel drugs tested as maintenance therapy.

\section{Key Points}

- The failure or success of HCT is significantly impacted by the patient's immune status.

- Harmonizing individualized drug monitoring during conditioning, graft design, and immune monitoring is key for patient surveillance and needs to be registered within the new cellular therapy registry of EBMT.

- A harmonization procedure to achieve a more balanced immune reconstitution might have a more profound impact on patient survival than any other novel maintenance therapy and allow for a better success rate for novel drugs tested as maintenance therapy. 


\section{References}

Admiraal R, van Kesteren C, Jol-van der Zijde CM, et al. Association between anti-thymocyte globulin exposure and $\mathrm{CD} 4+$ immune reconstitution in paediatric haemopoietic cell transplantation: a multicentre, retrospective pharmacodynamic cohort analysis. Lancet Haematol. 2015;2:e194-203.

Admiraal R, Nierkens S, de Witte MA, et al. Association between anti-thymocyte globulin exposure and survival outcomes in adult unrelated haemopoietic cell transplantation: a multicentre, retrospective, pharmacodynamic cohort analysis. Lancet Haematol. 2017;4:e183-91.

Bartelink IH, Lalmohamed A, van Reij EM, et al. Association of busulfan exposure with survival and toxicity after haemopoietic cell transplantation in children and young adults: a multicentre, retrospective cohort analysis. Lancet Haematol. 2016;3:e526-36.

Boelens JJ, Admiraal R, Kuball J, Nierkens S. Fine-tuning antithymocyte globulin dosing and harmonizing clinical trial design. J Clin Oncol. 2018;36:1175-6.

Czerw T, Labopin M, Schmid C, et al. High CD3+ and $\mathrm{CD} 34+$ peripheral blood stem cell grafts content is associated with increased risk of graft-versus-host disease without beneficial effect on disease control after reduced-intensity conditioning allogeneic transplantation from matched unrelated donors for acute myeloid leukemia - an analysis from the Acute Leukemia Working Party of the European Society for Blood and Marrow Transplantation. Oncotarget. 2016;7(19):27255-66.

Davids MS, Kim HT, Bachireddy P, et al. Ipilimumab for patients with relapse after allogeneic transplantation. N Engl J Med. 2016;375:143-53.

de Koning $\mathrm{C}$, Plantinga $\mathrm{M}$, Besseling $\mathrm{P}$, et al. Immune reconstitution after allogeneic hematopoietic cell transplantation in children. Biol Blood Marrow Transplant. 2016;22:195-206.

de Witte MA, Sarhan D, Davis Z, et al. Early reconstitution of NK and gammadelta T cells and its implication for the design of post-transplant immunotherapy. Biol Blood Marrow Transplant. 2018;24(6):1152-62.

Du J, Paz K, Thangavelu G, et al. Invariant natural killer T cells ameliorate murine chronic GVHD by expanding donor regulatory T cells. Blood. 2017;129:3121-5.

Finkelstein SE, Carey T, Fricke I, et al. Changes in dendritic cell phenotype after a new high-dose weekly schedule of interleukin-2 therapy for kidney cancer and melanoma. J Immunother. 2010;33:817-27.

Greco R, Ciceri F, Noviello M, et al. Immune monitoring in allogeneic hematopoietic stem cell transplant recipients: a survey from the EBMT-CTIWP. Bone Marrow Transplant. 2018;53:1201-5.

Keustermans GC, Hoeks SB, Meerding JM, et al. Cytokine assays: an assessment of the preparation and treatment of blood and tissue samples. Methods. 2013;61:10-7.

Locatelli F, Merli P, Pagliara D, et al. Outcome of children with acute leukemia given HLA-haploidentical HSCT after alphabeta T-cell and B-cell depletion. Blood. 2017;130:677-85.

Malard F, Labopin M, Chevallier P, et al. Larger number of invariant natural killer T cells in PBSC allografts correlates with improved GVHD-free and progressionfree survival. Blood. 2016;127:1828-35.

Mathew NR, Baumgartner F, Braun L, et al. Sorafenib promotes graft-versus-leukemia activity in mice and humans through IL-15 production in FLT3-ITDmutant leukemia cells. Nat Med. 2018;24:282-91.

Pasquini MC, Devine S, Mendizabal A, et al. Comparative outcomes of donor graft CD34+ selection and immune suppressive therapy as graft-versus-host disease prophylaxis for patients with acute myeloid leukemia in complete remission undergoing HLA-matched sibling allogeneic hematopoietic cell transplantation. J Clin Oncol. 2012;30:3194-201.

Perko R, Kang G, Sunkara A, et al. Gamma delta T cell reconstitution is associated with fewer infections and improved event-free survival after hematopoietic stem cell transplantation for pediatric leukemia. Biol Blood Marrow Transplant. 2015;21:130-6.

Scheper W, van Dorp S, Kersting S, et al. Gammadelta $\mathrm{T}$ cells elicited by CMV reactivation after Allo-SCT cross-recognize CMV and leukemia. Leukemia. 2013;27:1328-38.

Soiffer RJ, Chen YB. Pharmacologic agents to prevent and treat relapse after allogeneic hematopoietic cell transplantation. Blood Adv. 2017;1:2473-82.

Soiffer RJ, Kim HT, McGuirk J, et al. Prospective, randomized, double-blind, phase III clinical trial of antiT-lymphocyte globulin to assess impact on chronic graft-versus-host disease-free survival in patients undergoing HLA-matched unrelated myeloablative hematopoietic cell transplantation. J Clin Oncol. 2017;35:4003-11.

Open Access This chapter is licensed under the terms of the Creative Commons Attribution 4.0 International License (http://creativecommons.org/licenses/by/4.0/), which permits use, sharing, adaptation, distribution and reproduction in any medium or format, as long as you give appropriate credit to the original author(s) and the source, provide a link to the Creative Commons license and indicate if changes were made.

The images or other third party material in this chapter are included in the chapter's Creative Commons license, unless indicated otherwise in a credit line to the material. If material is not included in the chapter's Creative Commons license and your intended use is not permitted by statutory regulation or exceeds the permitted use, you will need to obtain permission directly from the copyright holder. 